\title{
A Review Paper on "Modeling of Traffic Simulation System for Dynamic Traffic with Multi Agent Framework"
}

\author{
Mr. Sushant Madhukar Gajbhiye ${ }^{1, \mathrm{a}}$, Dr. Arun Patel ${ }^{2, \mathrm{~b}}$ \\ ${ }^{1} \mathrm{PhD}$ Scholar, RKDF University, Gandhi Nagar Campus, Bhopal (MP), India. \\ ${ }^{2}$ Professor, RKDF University, Gandhi Nagar Campus, Bhopal (MP), India.
}

\begin{abstract}
Traffic in developing and developed countries mostly having heterogeneous traffic conditions. It comprises with wide range of physical dimension of vehicles and its weight. Due to dynamic traffic it causes series stain on road infrastructure and also effect the road condition (Structure \& Surface condition), this affect the increasing the road accident. At intersection point due to heavy traffic jam density increases and it reflect the increasing of travelling time (Time \& Distance Headway) for the driver it also affect the total cost of the trip as the vehicles always starting in position during jam occur at intersection. For better traffic movement traffic simulation need to be implemented for distribution the traffic lane wise or by size of the vehicles.

This article focuses on the study and modeling of dynamic traffic using multi-agent framework. In this review paper different keywords have been consider by every individual researcher, which can be useful for the improvement of traffic condition and reduction of accidents at intersection point or at crossing. There are different types of modal used by the researcher like single regime linear modal, dual regime modal, polynomial modal, exponential modal for the distribution of traffic by considering vehicular characteristic on and geometric design. Intelligent transport can be used for the improvement of traffic condition and it uses the multi agent can be maintained or utilized according to the traffic conditions.
\end{abstract}

Keywords: Speed, Acceleration, Deceleration, Driver Behaviour, Geometric Design, Vehicle characteristics.

\section{INTRODUCTION}

The traffic is the basic aspect which the person is used in his daily life. There are different types of trip like (Home, Office, Enjoying, Work etc.) the person use in his routing. It is his necessity to achieve his goal. Vehicles of different category used for transportation according to the driver convenience and safety. Heterogeneous and homogeneous traffic conditions are generally occurring according to their traffic intensity and moving capacity having speed limit vary from $30-100 \mathrm{~km} / \mathrm{hr}$ due to which the deceleration and acceleration behaviour is also different due to varying of physical properties, deceleration, speed, acceleration, driver behaviour etc. Dynamic traffic occur on street in which vehicles move individually on road width available and follow the different level of speed limit. Vehicles having smaller dimension utilize it benefits and vary its speed according to their convenience but the heavy vehicles need to reduce its speed and need to follow the rules and regulation.
The study of regulatory system, intersection, single intersection, intensity of traffic behaviour of different type of vehicles is required for realistic simulation and modeling of traffic streams as their acceleration and deceleration behaviour varies over a wide range depending upon their power to weight ratios. Further, acceleration/deceleration pattern of driver may also depend on his/her characteristics like age, driving experience, qualification and financial status etc. Effect of driver behaviour on speed, acceleration and deceleration needed higher understanding of driver belief and attitude towards speeding. Speeding, acceleration and deceleration behaviour of drivers also depends on road attributes, such as lane width, number of lane, horizontal and vertical alignment curves and pavement performance. Higher performance vehicles equipped with advanced system also affect the driver speeding, acceleration/deceleration behaviour of vehicles. Estimation of driving behaviour with regard to speeding pattern can also be assessed by actual observation of driver speed under various set of condition like road classification and different traffic condition.

Therefore, an attempt has been made to study the multi agent framework \& its behaviour for different type of vehicles (like trucks, Bus, auto-rickshaw, cars, bike, HMV \& LMV). Further the effect of driver characteristics (like education, age, driving experience etc.) on their acceleration and deceleration behaviour has been also explored.

\section{LITERATURE REVIEW}

\section{Researcher Used The Single Regime Linear Model}

Loutzenheiser ${ }^{[13]}(\mathbf{1 9 3 8})$ conducted a study on acceleration and speed of vehicles for finding the correlation between them. He considered six to eight light weight vehicles for data collection. Data collected for three years (1935-1937) having speed $48.30 \mathrm{kmph}$ and above. For analysis number of trails taken for highest acceleration having differing in drivers then cumulated all necessary data to gent increment in speed of $5 \mathrm{mph}$. he found highest acceleration of 1.74 $\mathrm{m} / \mathrm{sec}^{2}$ at vehicle having speeding vary from 0 to 8.05 $\mathrm{kmph}$ and rate downfall straight to $0.45 \mathrm{~m} / \mathrm{sec}^{2}$ at speed 60 to $70 \mathrm{mph}$. From research he cumulated that a straight falling model was more suitable for acceleration and speed accumulation. 
Beakey $^{[3]}$ (1938) studied on Characteristics of Deceleration and Acceleration of low motor vehicle of private vehicle (passenger car), same concept what Loutzenheiser ${ }^{[13]}$ analyze. In his study data collection was done in two parts one in 1931 and other in between 1935 to 1937. A series of test was conducted on a passenger car and data collected by using manual transmission in the form of speed. Result shows separately for individual vehicle having increment in speed $16.1 \mathrm{kmph}$ acceleration is measured in two parts, 1) Full-throttle acceleration in second gear, 2) Higher gear acceleration at Full-throttle without considering standard acceleration to stopping. The maximum overall vehicular acceleration was 42.3-64.4 $\mathrm{kmph}$ for optimum vehicular speed for shifting from dual to higher gear. It observed that an approximately line fall to zero at highest speed for every individual vehicles in year 1935-37, but at the year 1931 it found that the acceleration for low motor vehicle was near about 0.4 meter per square second below the average vehicular speed. His conclusion was found same as Loutezenheiser $^{[13]}$ specified that the accumulation of average acceleration of every vehicle was same.

Bellis $^{[4]}$ (1960) propose the model, linearly decreasing acceleration model. Data collection was done at three signalized intersection for two types of vehicles such as, passenger car and truck (single unit truck). He identified that the heavy motor vehicle (Truck) have 12.8 meter average length which separate current heavy vehicles were bigger and having more carrying weight capacity. New Jersey data was collected at different intersection point (signalize intersection) by making group of five people for pressing and holding the telegraph switch wire key to a 20 percent graph recorder. The data was collected is not useful for finding the acceleration profile because a few traps were carried out at different sites but it helps to determine the cumulative travel time of in site at six different position. The collected raw reading for heavy motor vehicle (HMV) was insufficient and not able to help in determining the acceleration of vehicle or for analysis purpose, but the data was collected for the truck is complete and to be useful for analysis purpose. The result shows that the analysis have few gaps to fill but helps to make a decision that shows a strongly straight falling relation between vehicles $(\alpha=1.6 \& \beta=0.12)$. So regarding to the value, the suitable model was proposed was linearly decreasing acceleration model with respect to speed.

Evans $^{[8]}$ (1981) researched on queued vehicles to elaborate the Inter correlation between speed and acceleration. He analyzed 15138 queued vehicles (motorist) data of Detroit city near suburban area and used sensors for data collection. Queued vehicles (Bikers) stopped at signalized intersection. At certain distance sensors was used where the vehicular speed reached the maximum acceleration 35-55 kmph with further no stopping point (Zero speed). This data is useful to carry out the maximum acceleration at starting of vehicles where the signalized intercept occurred. Analysis shows that there was slightly increased of acceleration $0.1 \mathrm{~m} / \mathrm{s} 2$ in left side lane compare to right lane at different position where vehicles are queued. He proposed a straight decreasing model where the bikers cannot overtake the other queued vehicles as they are behind each other at stopping point. This type of model helped for the designing of signalized intersection point where heterogeneous traffic always occurs.

Bonneson $^{[5]}$ (1992) followed Rothery ${ }^{[8]}$ for his research study. He used same technique and method applied by Evan but the distance between stopping point and sensors were quite less compared to Rothery ${ }^{[8]}$ did. The same experimental setup was done for finding the acceleration \& speed data. A 4820 vehicles (passenger car) data was collected at different five intersecting points (signalized intersection) in different states. For the analysis the cumulative raw data (averaged) was considered and by analyzing he proposed an effective line correlation between vehicular speed and its acceleration with $a=2.02$ for separate stream of traffic. He got same reading at other five locations however reading varies from $0.1184-0.1326$. This analysis is somewhat nearer to Beakey ${ }^{[3]}$ as well. He proposed decreasing falling linearly model with correlation of speed and acceleration.

Collins $^{[6]}$ (1999) focused on speed designing and evaluating method with respect to alignment by considering speed simulation model. He developed cumulative speed model at different scenario i.e. simple, horizontal, compound, vertical, reverse and combined curves by evaluating expected speed of different village roads. Speeding model used for evaluating the consistence occurrence of facility provided to gent (generate) a speeding model towards the road alignment. The required speed cannot be accumulated as the vehicular speed analyzed on tangential manner without considering of vertical alignment and horizontal alignment act as a speed desired on road. He collected data of six different states of 176 rural roads in tangential manner (Two Lane) varying speed 93-104 $\mathrm{kmph}$ with $85^{\text {th }}$ percentage of speed cumulated. Speed considered (predicted) using three different methods i.e. assuming designing speed, equation of prediction of speed and equation of TOPAS were accumulated and minimum (lowest) speed was considered for designing purpose. For designing cumulative speed model he considered speed module before and after the movement of vehicles on curve. For accumulation driver need to maintain the desired speed if it is less then driver reduced tis acceleration and after covering the tangential lane (curve) he accelerate. The conclusion was made that the speed always vary compared to desired speed, to match the level of consideration a limit is set $60-100 \mathrm{kmph}$ at a 100 meter horizontal curve. The model suggested by him is suitable for designing of IHSDM module.

Gary ${ }^{[10]}(2000)$ conducted the analysis on acceleration and deceleration of different category of vehicles to verify whether the vehicles were starting from zero (at rest) or not. Previous study suggested some point that helps modeling of vehicular acceleration and deceleration. It represent straight falling model of speed related to vehicular acceleration that helps modeling of vehicular 
acceleration and deceleration. It found that the different acceleration value more useful for designing compared to constant (average) value. Low speed data collected or starting of vehicle data collected. The accumulation of acceleration straight model was very low speed at starting point as the data was not preciously accurate but it does not affect the travel distance as the speed was nearly zero. The value required for designing purpose based on highest acceleration. At stopping highest acceleration adopted by the driver at very low speed to achieve more distance. At low speed design vale and practical value seems to conflict as it depend of driver assumption. He concluded that there is a variation in speed data and one cannot directly say that the initial value had no effect on data. One can have different types of model that shows the excellent data fit.

\section{RESEARCHER USED THE SINGLE REGIME POLYNOMIAL MODEL}

Dockerty $^{[7]}$ (1966) conducted research on vehicular characteristics of moving vehicles. An actual motorist data collected to analyses it behaviour. Data was collected for all types of vehicles such as passenger car, at low, medium and high motor vehicle and analyze each type of vehicle separately. For the study of acceleration characteristics he specially collected data of queued vehicle at London for two different signalized intersection point using motion picture photography in England. He separated the data for analysis purpose and measured the vehicle distance at one second intervals. His result somewhat different compared previous researcher study, it did not follow the straight falling model with $a=2.27$ and $b=0.1487$. Results represent that forward vehicle accelerate slowly with $\mathrm{a}=1.88$ and $b=0.120$. His result matches to Beakey ${ }^{[4]} \&$ Belis $^{[3]}$ concluded. He concluded that the straight falling model does not fit for designing of vehicular acceleration as the value vary high to low.

Akcelik $^{[1]}$ (1987) studied a acceleration model on different traffic conditions, collected data from Australia at three different location area (urban, central and non-urban). A raw data of 1037 count has been collected from stopping position using instrumented car. He proposed the polynomial curve that satisfies the zero jerks at starting point and at end point where smooth acceleration required. For analysis purpose he collected the time headway data of second by second intervals. A nineteen measurement data points has been captured for thousands of vehicles having different driver at different location of $956 \mathrm{~km}$. Data accumulation shows that the polynomial data model was best suited for change in acceleration. His result matches the Dockerty ${ }^{[7]}$ result where the pedestrian interference occurred.

Aycin $^{[2]}$ (1998) studied a straight acceleration algorithm with continuous model without considering step wise acceleration. He considered LMV (car) tracing algorithm for homogeneous and heterogeneous traffic condition to validate its model. He analyses driver breaking time and computed with independent time factor. The data collected by him shows a strong correlation in between field data and simulated data. Different model observed in texas, acceleration behaviour of driver is taken at different location where a stopping point was not constant this behaviour shows a straight falling model. Acceleration driver behaviour was also observed where the desired value explained by straight acceleration model. Here the time evaluation factor is considered at every step for simulation of traffic. The approach of Benekohal ${ }^{[2]}$ were validated on homogeneous and heterogeneous traffic of new LMV (car) tracing behaviour. In real time accumulation (simulation) of car tracing model used step and constant acceleration model which affect real and erratic behaviour. Driver break time (reaction time) was considered for simulation of traffic without affecting simulation time. His data was best suited for single regime polynomial model.

Hallmark $^{[11]}$ (1998) worked on speed distribution at signalized intersection he used the simulation model that helps to predict the problem occurred at intersection point that also helps for comparing the field data with simulation model where the data collected at signalized intersection. The simulation model is more suitable for comparing different traffic condition having different time intervals for separated vehicles. He found highest acceleration rate by analyzing straight speed relation with acceleration having velocity zero at highest acceleration and highest velocity at zero acceleration. For data collection laser range finder (LRF) was used at intersection point where signals are available. Laser gun have a frequency of $238.4 \mathrm{~m} / \mathrm{s}$ accuracy with $15.24 \mathrm{~cm}$ capturing. NETSIM is used for data simulation. It predicted the greater fraction point compare to field data at actual intersection. He suggested that NETSIM is suited for calibration of data and it proved that it is suited for modeling purpose.

By accumulating reviews of all literature it concludes, most of the studies propose a single regime linear model for acceleration behaviour of vehicle. Some of the researchers like Dockerty ${ }^{[7]}$ (1966), Akcelik ${ }^{[1]} \&$ Biggs $^{[1]}$ (1987), Mark Besley (2002) proposed a single regime polynomial model for vehicle acceleration. Most of these studies are limited to study of acceleration characteristics of LMV \& HMV (Cars \& Trucks). This motivate author to conduct a detailed study on acceleration trajectory and deceleration trajectory behaviour of different category of vehicles.

\section{DRIVER BEHAVIOUR}

Fildes $^{[9]}$ (1991) in Monash University undertook the study of driver behaviour to speeding and explain that drivers above age 55 were excessively slow travelers and the drivers above age 34 were excessively high speeder. These observations were true for all the road geometry (straight and curved). It is also concluded that sex of the driver was not associated with speeding.

Poe C.M ${ }^{[15]}$ (1996) reported that gender, number of passenger and passenger vehicle type were not much significant aspects affecting speed but senior drivers traveled about $2 \mathrm{~km} / \mathrm{h}$ less than young drivers. 
Kang $^{[12]}$ (1998) studied the driver speeding in Korea, taking into account factors such as personal, vehicle, attitudinal and trip characteristics. Male drivers with higher incomes tend to drive faster as compared to drivers with less income. Experienced drivers drove faster and trip distance and frequent road use were also important factors in choosing speed.

Mehmood $^{[14]}$ (2009) studied driver speeding behaviour on roads of Al Ain (United Arab Emirates). The data was collected based on the questionnaire designed for the research. Data was collected for three years from 2005 to 2007 in two main sections, one having demographic data like gender, age, nationality, status of knowledge (education), site selection, vehicle category, experience in driving, and other data regarding attitude on five different points like its feature, behaviour, accumulation, grasping, competent from strongly agree and disagree. About 650 drivers were selected to respond out of which $67 \%$ were male and $33 \%$ were female. Authors concluded that as drivers mature through age and develop sense of responsibility and lower the speed of vehicle. However what is the effect of lowering the speed on acceleration and deceleration is not reported in the study.

By accumulation of reviews of all literature it graphed (observed) that, driver behaviour always affect the traffic regulatory system. Different keywords were used for the study like age, sex, income, qualification, driving experience, eyesight, human structure etc. for the study.

\section{CONCLUSION}

From the study of many research papers, different conclusion has been given by the researchers,

Speed, Acceleration \& deceleration, vehicular characteristics of vehicles and driver behaviour were require for designing of transportation module and geometric of road that involves direction, flow, driver ways, traffic signal, changing bias (turning), intersection point, crossing and software used for simulation purpose. Therefore, study has been conducted and analyzed to do further research for reducing the jam density at intersection. Effect of driver's attributes (like age, driving experience, education and income etc) is also investigated on their acceleration and deceleration behaviour. Some basic conclusions were drawn from the previous research work.

Acceleration of vehicles (value) initially reached at highest peak maximum value at lower speed range and later on acceleration value reduced decreases with increasing of speed.

Deceleration values generally increases to maximum value (in magnitude) at lower speed range and later on deceleration value decreases (in magnitude) with increasing of speed.
The dual regime model is found more suitable to specify the vehicular acceleration for entire speed range than single regime model. Present study evaluates dual regime linear model and dual regime linear-exponential model found satisfactorily implementable.

In vehicular deceleration maneuver, dual regime linear model and dual regime linear-exponential model is evaluated and observed and it is found that both of these models explain the deceleration behaviour of vehicle satisfactory implementable.

\section{REFERENCES}

[1] Akcelik, R. \& D.C. Biggs (1987). "Acceleration Profile Models for Vehicles in Road Traffic". Transportation Science, Vol. 21, No. 1 pp. 36-54.

[2] Aycin, M., Benekohal, R. (1998). "A Linear Acceleration CarFollowing Algorithm for Autonomous Intelligent Cruise Control Systems". In Proceeding, 5th International Conference on Applications of Advanced Technologies in Transportation Engineering. Vol. 1678, pp. 116-127.

[3] Beakey, John (1938). "Acceleration and Deceleration Characteristics of Private Passenger Vehicles". Highway Research Board Proceeding, Vol. 18, pp. 81-89.

[4] Bellis, W.R (1960). "Capacity of Traffic Signals and Traffic Signal Timing”. Highway Research Board Proceeding, National Research Council, Washington, D.C., pp. 45-67.

[5] Bonneson, James A. (1992). "Modeling Queued Driver Behavior at Signalized Junctions". Transportation Research Record 1365, TRB, National Research Council, Washington, D.C., pp. 99-107.

[6] Collins, J., Fitzpatrick, K. (1999). "Speed Prediction for Two-Lane Rural Highways". Transportation Research Board, Volume 1737 / 2000, U.S.

[7] Dockerty, A. (1966). "Accelerations of Queue Leaders from Stop Lines". Traffic Engineering and Control, Vol. 8, No. 3, pp. 150155.

[8] Evans L. \& Rothery R. (1981). "Influence of Vehicle Size and Performance on Intersection Saturation Flow". Proc., 8th International Symposium on Transportation and Traffic Theory, University of Toronto Press, Toronto, Ontario, pp. 193-222.

[9] Fischer, A. (1997). "Exploitation level of maximum deceleration in motorcycle braking tests (in German)". MS-Thesis, Vienna University of Technology, Vienna, Austria.

[10] Gary Long (2001). "A Policy on Geometric Design of Highways and Streets," Fifth edition of AASHTO's "Green Book" 2004.

[11] Hallmark, S \& Guensler, R. (1998). "Stop line Distributions of Speed and Acceleration for Signalized Intersections". Presented at 91st Annual Meeting of the Air and Waste Management Association, San Diego, Calif.

[12] Kang, K. (1998) "Ordered Probit Model of the Speed Selection Behavior", Results Based on a Korean Micro Data. Transportation, Traffic Safety and Health Human Behavior, Fourth International Conference, Tokyo, Japan. Publication AJD-18.

[13] Loutzenheiser, Donald W. (1938) "Speed change Rates of Passenger Vehicles". Highway Research Board Washington DC. Proceeding, Vol. 18. Page 90-99.

[14] Mehmood Arif (2009) "Determinants of Speeding Behavior of Drivers" in Al Ain (United Arab Emirates) Journal of Transportation Engineering, Vol. 135, No. 10, October, pp. 721729.

[15] Poe, C.M., Tarris, J.P., and Mason, J.M. (1996) "Inuence of Access and Land Useon Vehicle Operating Speeds Along Low-Speed Urban Streets". Conference Proceedings for the Second National Access Management Conference, Vail, Colorado. pp. 46-54. 